\title{
British Sleep Society: the COVID-19 pandemic response
}

\author{
Joerg Steier ${ }^{1,2,3}$, Simon Durrant ${ }^{1,4}$, Alanna Hare ${ }^{1,5}$; on behalf of the BSS Executive Committee \\ ${ }^{1}$ British Sleep Society, Lichfield, UK; ${ }^{2}$ Sleep Disorders Centre, Guy's \& St Thomas' NHS Foundation Trust, London, UK; ${ }^{3}$ Faculty of Life Sciences \\ and Medicine, King's College London, London, UK; ${ }^{4}$ Lincoln Sleep Research Centre and School of Psychology, University of Lincoln, Lincoln, \\ UK; ${ }^{5}$ Royal Brompton \& Harefield NHS Foundation Trust, London, UK \\ Correspondence to: Joerg Steier, FRCP, MD, PhD. Guy's \& St Thomas' NHS Foundation Trust, Westminster Bridge Road, London SE1 7EH, UK. \\ Email: joerg.steier@gstt.nhs.uk.
}

Submitted Jun 29, 2020. Accepted for publication Jul 18, 2020.

doi: $10.21037 /$ jtd-2020-059

View this article at: http://dx.doi.org/10.21037/jtd-2020-059

\section{British Sleep Society (BSS) webinar}

The current pandemic caused by a novel coronavirus, named COVID-19, holds the entire world to ransom. A proportion of the infected patients becomes critically ill, with millions being infected and hundreds of thousands who have died so far. In some countries, national lockdown restrictions are being slowly lifted, but the World Health Organization (WHO) still registers increasing numbers of confirmed COVID-19 infections across its membership states.

Against this background, the BSS brought together doctors from the respiratory and critical care response teams in Wuhan, China and London, UK in a webinar to exchange their knowledge and expertise and discuss current best practice in the management of patients with COVID-19. Following their presentations, sleep experts from the Executive Committee of the BSS discussed the impact of the pandemic and lockdown on sleep and shared practical advice regarding sleep health.

\section{Respiratory and critical care response to the COVID-19 pandemic}

Professor Fengming Luo and Dr. Wanhong Yin from the West China Hospital of Sichuan University, China were involved in the direct respiratory and critical care response team during the outbreak of the pandemic in Wuhan. They gave an overview about the logistics, the medical and infrastructure measures taken and the difficulties that they had to overcome facing this novel virus. During the outbreak, it became clear that the virus did not only affect the lungs, but also the cardiovascular, renal and neurological systems. The Chinese workforce developed protocols for the use of personal-protective-equipment (PPE; Figure 1), prevention of infection, clinical practice and testing-andtracing.

Professor Nicholas Hart from Guy's \& St Thomas' NHS Foundation Trust (GSTT) in London then spoke about the NHS Response to COVID-19 in the London area. Prof. Hart is the Clinical Director for Pulmonary, Adult Critical Care and Sleep (PACCS) and as such was involved in the South London Respiratory and Critical Care Network planning during the COVID-19 pandemic, the area most hit by the pandemic in the UK (1). The first patient with COVID-19 at GSTT was admitted to intensive care on the $3^{\text {rd }}$ March 2020 and, following a better understanding of the disease, triggered a comprehensive integration between acute medicine, anaesthesia, infectious diseases, respiratory and critical care that allowed for a rapid expansion of hospital capacity (Figure 2). Surgical elective interventions were reduced and a planned expansion of the critical care area to 234 critical care beds was initiated. The redeployment of staff required training of some 1,550 clinical and many more non-clinical staff, accessing education and online training and videos; this included 245 junior and senior doctors and 473 nurses. Critical Care, Respiratory, Thrombosis and Renal Pathways were established, 'lines' and 'intubation' teams, as well as 'proning' teams started to work non-stop. Virtual communication 'lifelines', were initiated with families and next of kin to allow to them to virtually "visit" their loved ones via videocalls. Ventilator and equipment procurement were coordinated for South London and data analytics support was provided centrally. 


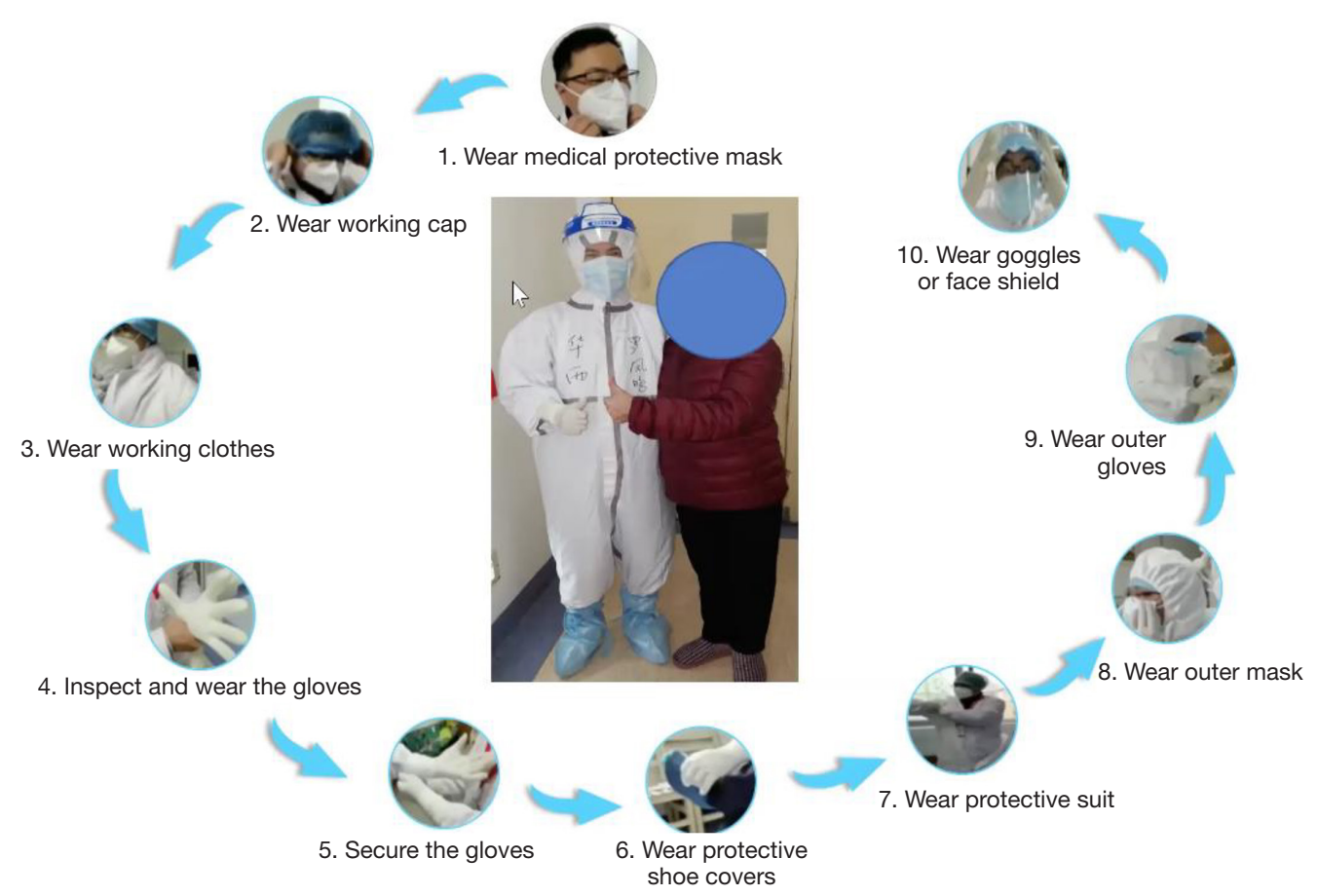

Figure 1 "Donning" procedure of the Chinese Wuhan response team.

South London saw the peak of the first wave in the first half of April with steadily declining numbers of critically ill patients thereafter. Critical care bed capacity was not breached: at the peak there were 130 patients at any given time under the care of GSTT. This compares to a planned critical care bed capacity of 99 beds for the winter season with influenza.

\section{Pathophysiology}

Profs Luo, Yin and Hart described how, in the first week of the infection, patients typically experience a viral pneumonia, with subsequent weeks causing an increased drive to breathe, acute lung injury, acute cardiac and skeletal muscle injury, possible kidney and liver injury and intestinal involvement. There is some suggestion of two phenotypes of acute respiratory distress syndrome (ARDS): type 'L' describes patients with low elastance, high compliance and low V/Q ratio; type ' $\mathrm{H}$ ' includes patients with high elastance and low compliance $\left(<30 \mathrm{~mL} / \mathrm{H}_{2} \mathrm{O}\right)$ and high right-to-left shunt (2). Neural respiratory drive was generally high due to the increased work of breathing, high transpulmonary pressures and shearing forces, and this was complicated by worsening pulmonary oedema and, over time, developing fibrotic changes. Closing capacity of the airway was often reached, particularly in obesity, with microtrauma worsening the oedema with every breath. An increased positive end-expiratory ventilation pressure (PEEP) during ventilation was found to be helpful to avoid this and recruit lung volumes. Pulmonary vessels were exposed to significant hypoxic vasoconstriction, due to the Euler-Liljestrand effect, and acute pulmonary hypertension developed.

\section{Post-critical care follow up}

Prof. Hart explained that in patients who were discharged from critical care at GSTT, about $50 \%$ of patients underwent regular post critical care rehabilitation, $25 \%$ required complex respiratory and $25 \%$ complex renal support; $70 \%$ of tracheostomised patients survived, about $10 \%$ of tracheostomised patients died, and $30 \%$ had weaned successfully by the time of the webinar. Post critical care follow up at 4-6 weeks included 1,136 patients with a mortality of $19 \%, 7 \%$ of the patients still remaining inpatients at that time. However, half of the patients in the follow up clinics had returned to their pre-COVID-19 baseline. The most common pathologies identified at 


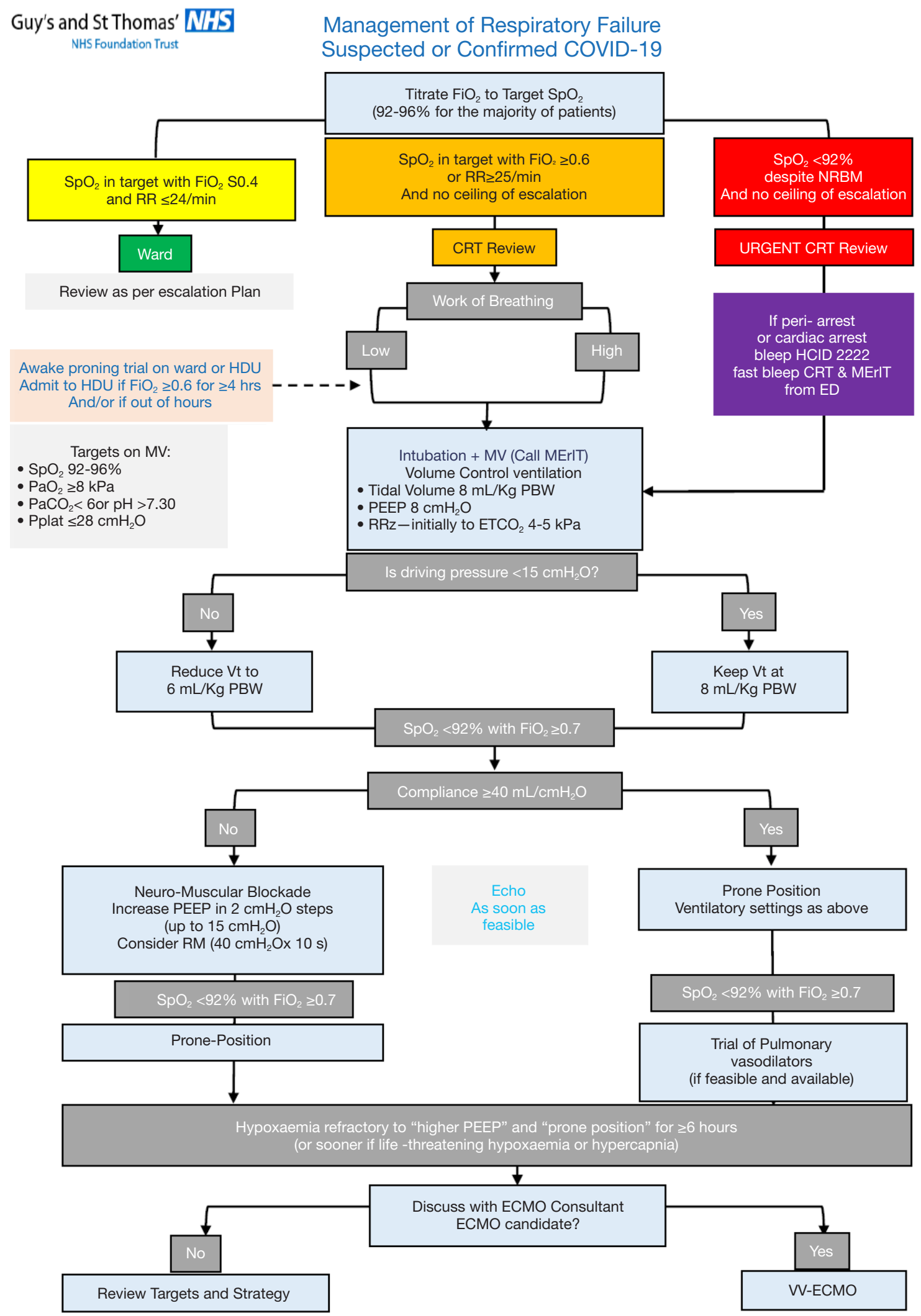

Figure 2 Respiratory and critical care treatment algorithm at Guy's \& St Thomas' NHS Foundation Trust, London, UK. 
follow up included interstitial lung disease (ILD), including organising pneumonia, cardiac and neuropsychiatric issues.

\section{Insomnia during COVID-19}

In the second part of the webinar, the focus was on the impact of COVID-19 on sleep and Professor Jason Ellis spoke on insomnia during the pandemic. Referencing surveys of the public exploring the mental health and sleep impacts of COVID-19 from both China (3) and Italy (4), Professor Ellis opened by noting that the published data suggest that the public worldwide has experienced a major mental health burden as a result of the pandemic. In both papers, a significant proportion of respondents reported that their sleep quality had been affected as a result of the pandemic, with $57 \%$ of respondents in the Italian survey reporting poor sleep quality, as well as high levels of anxiety and distress. Both papers found that younger participants were more likely to report sleep disturbance, whilst the Italian study also found that female sex, fear of contact with cases of COVID-19 and uncertainty around COVID-19 infection increased the likelihood of impaired sleep.

Professor Ellis proposed a diagnostic for acute insomnia comprised of a "trigger" to the sleep disturbance, which could be any life event or train of life events, resulting in a significant reduction in quality of life, coupled with distress at the current situation. The sleep disturbance should occur for at least 3 nights per week and last between 3 days and 3 months (following which the disorder is better characterised as chronic insomnia). The severity of acute insomnia can be subjectively assessed by the patient as mild, moderate or severe, or quantitively as a sleep onset latency and/or wake after sleep onset greater than or equal to 30 minutes (5).

Moving to management, Professor Ellis asked "can we circumvent the transition from acute to chronic insomnia with a 'single-shot' of CBT-I?". Although cognitive behavioural therapy for insomnia (CBT-I) is well-established for the treatment of chronic insomnia, it has been less well-studied in acute insomnia. In addition, access to CBT-I is challenging in many areas and countries and is often considered too burdensome to perform in primary care where, one might argue, it is most likely to be beneficial. In a community-based pragmatic parallel group randomized controlled trial, forty adults with acute insomnia were given either a single 60- to 70-minute session of CBT-I $(n=20)$, with an accompanying self-help pamphlet, or were wait listed as the control group $(n=20)(6)$. All subjects were offered a full individual course of CBT-I on completion of the study, regardless of group allocation.

The major components of the "single shot" CBT-I were sleep hygiene advice and sleep education to identify and address sleep related dysfunctional thinking; the principles and practice of sleep restriction and a pamphlet advising on the principles of stimulus control, cognitive control, and the use of imagery distraction techniques.

At follow-up, there was a significant difference in Insomnia Severity Index (ISI) scores between those in the CBT-I group and those in the control group [ $\mathrm{t}(38)$ $=2.24, \mathrm{P}<0.05]$. Using the criteria of an ISI score $<10$ being indicative of insomnia remission, at follow-up 12 of 20 participants $(60 \%)$ in the treatment group had remitted compared to 3 of $20(15 \%)$ in the control group. This difference in cases at follow-up was significant $\left(\chi^{2}=8.64, \mathrm{df}\right.$ $=1, \mathrm{P}<0.003)$, indicating that this brief CBT-I intervention is sufficient to elicit significant improvements in sleep in individuals with acute insomnia. In a further study, the authors showed that "single shot" group treatment is as efficacious as individual treatment (7).

Concluding, Professor Ellis acknowledged that the COVID-19 pandemic has had a significant impact on individuals' sleep worldwide and, in the face of an increasing incidence of acute insomnia, we must look for effective treatment strategies which can be rapidly deployed, with a view to circumventing progression to chronic insomnia and its attendant impacts on individuals' mental health. "Single shot" CBT-I may provide a pragmatic solution in these challenging times.

\section{BSS statement on sleep-related advice during the COVID-19 pandemic}

The last talk in the webinar, delivered by Dr. Simon Durrant, Treasurer of the BSS, focused on another key part of the response to the pandemic from a sleep perspective: the release of the COVID-19 Statement on sleep during the pandemic by the BSS on $17^{\text {th }}$ April 2020. The decision to release a statement was taken in light of increasing evidence of COVID-19-related sleep problems, together with uncertainty over whether or not patients with obstructive sleep apnoea (OSA) should continue with CPAP treatment during the pandemic $(8,9)$. The talk reflected the four parts of the statement, which first outlines general government advice on hygiene and what to do in the event of an infection, before moving on to advice specific to sleep.

Clarity around treatment for OSA is especially important during the pandemic due to the additional problems 
patients with existing breathing disorders may experience should they contract COVID-19. The BSS position had previously been agreed as a signatory to the OSA Alliance statement (10), which suggests continuing with treatment in the absence of infection but temporarily stopping if infected, unless self-isolation in a different bedroom can be ensured and the treatment does not worsen symptoms. In all cases, communication (remotely) with service providers is recommended.

There have been increasing anecdotal reports of insomnia during the lockdown, which is perhaps not surprising in view of the effects of the pandemic: lockdown with reduced exercise opportunity, increased alcohol sales, more electronic device usage and heightened stress and anxiety around both the pandemic and concerns over jobs and livelihoods. Obtaining sufficient daily exercise is important for sleep quality as well as general health so it is important that any statement contains this. Similarly, alcohol consumption may shorten sleep onset latency, but does so at the expense of greater time awake after sleep onset and shortened REM sleep duration leading to standard advice to avoid alcohol consumption in the hours leading up to bedtime (11).

One key characteristic of the global lockdown has been a substantial increase in the use of electronic devices for communication. This has had many benefits but brings with it the risk of increased blue light exposure during the evening, leading to suppression of melatonin production and problems with sleep. Part of the statement focuses on this and suggests using low blue light mode and filters where possible.

Stress and anxiety play a large part in many-perhaps even most - cases of insomnia and in the current situation these are both greatly heightened. The last part of the BSS statement addresses these underlying causes and their impact on sleep quality. Modern therapeutic techniques combined with innovative technology are here to yield two potential solutions. Mindfulness aims to help people take a step back and reconnect with their thoughts and feelings more reflectively and apps are freely available to help with this, including programmes targeting sleep specifically. Meanwhile, CBT-I is available through electronic delivery as well as directly by a therapist and has proven effectiveness in treating insomnia (12). The European CBT-I Academy have released more detailed advice for sleep in the context of COVID-19 (13).

The BSS statement is designed to be helpful by addressing sleep problems and underlying causes specific to the current pandemic. If we can help people to sleep better, with the known benefits for immune function, we might yet help to improve quality of life in the general population.

\section{Take-home messages}

The unprecedented challenge of COVID-19 has forced clinicians across the world to redeploy whole workforces, rapidly expand critical care bed capacity, develop new working practices and institute strict infection control measures across entire hospitals within a matter of days (14). This has had to take place against a background of developing understanding about the pathophysiology of this complex infection, which involves multiple organ systems and in particular targets the respiratory system, resulting in a form of acute respiratory failure which has been striking in the variability of its presentation.

In addition to the millions who have experienced the direct physical effects of the infection, there are more individuals who have experienced indirect effects of the pandemic lockdown, which has led to widespread anxieties about personal and family members' health, finances and job security. These anxieties frequently affect individuals' sleep quality and quantity, with resultant impacts on daytime functioning and physical and mental health. The BSS have started a National Early Detection Screening survey (NEDS) for sleep associated problems. To date, 611 participants have submitted a response and of those, about $3 / 4$ noticed that their sleep had changed during the pandemic lockdown. More than half of the respondents experienced disrupted sleep, more than forty percent had difficulties falling or staying asleep, while about a quarter felt excessively sleepy or developed nightmares. Sleep hygiene advice, self-management resources (online) and guidance around sleep routines can improve these problems; the BSS has therefore published its statement on sleep-related advice during the COVID-19 pandemic containing guidance for the general population (15).

The BSS was honoured to play its part in bringing expertise and the global medical community together to share their knowledge and experience and further the understanding of this complex infection and its impacts on society.

\section{Acknowledgments}

We are grateful for the support of the educational subcommittee of the BSS in running the $1^{\text {st }}$ BSS webinar. 
Special thanks to Dr. Lizzie Hill, Professor Jason Ellis, Dr. Tim Quinnell, Professor Fengming Luo, Dr. Wanhong Yin and Professor Nicholas Hart who spoke or chaired the sessions. The views expressed are those of the authors and not necessarily those of the NHS, the NIHR or the Department of Health.

Funding: Professor Steier's contribution was partially supported by the National Institute for Health Research (NIHR) Biomedical Research Centre based at Guy's and St Thomas' NHS Foundation Trust and King's College London.

\section{Footnote}

Provenance and Peer Review: This article was commissioned by the editorial office, Fournal of Thoracic Disease for the series "Sleep Section". The article did not undergo external review.

Conflicts of Interest: All authors have completed the ICMJE uniform disclosure form (available at http://dx.doi. org/10.21037/jtd-2020-059). The series "Sleep Section” was commissioned by the editorial office without any funding or sponsorship. JS serves as an unpaid editorial board member of Fournal of Thoracic Disease. The authors have no other conflicts of interest to declare.

Ethical Statement: The authors are accountable for all aspects of the work in ensuring that questions related to the accuracy or integrity of any part of the work are appropriately investigated and resolved.

Open Access Statement: This is an Open Access article distributed in accordance with the Creative Commons Attribution-NonCommercial-NoDerivs 4.0 International License (CC BY-NC-ND 4.0), which permits the noncommercial replication and distribution of the article with the strict proviso that no changes or edits are made and the original work is properly cited (including links to both the formal publication through the relevant DOI and the license). See: https://creativecommons.org/licenses/by-nc-nd/4.0/.

\section{References}

1. Intensive Care National Audit \& Research Centre (ICNARC). ICNARC report on COVID-19 in critical care 22 May 2020. Available online: www.icnarc.org

2. Marini JJ, Gattinoni L. Management of COVID-19
Respiratory Distress. JAMA 2020. [Epub ahead of print].

3. Huang $\mathrm{Y}$, Zhao N. Generalized anxiety disorder, depressive symptoms and sleep quality during COVID-19 outbreak in China: a web-based cross-sectional survey. Psychiatry Res 2020;288:112954.

4. Casagrande M, Favieri F, Tambelli R, et al. The enemy who sealed the world: Effects quarantine due to the COVID-19 on sleep quality, anxiety, and psychological distress in the Italian population. Sleep Med 2020. [Epub ahead of print].

5. Ellis JG, Gehrman P, Espie CA, et al. Acute insomnia: current conceptualizations and future directions. Sleep Med Rev 2012;16:5-14.

6. Ellis JG, Cushing T, Germain A. Treating Acute Insomnia: A Randomized Controlled Trial of a "Single-Shot" of Cognitive Behavioral Therapy for Insomnia. Sleep 2015;38:971-8.

7. Boullin P, Ellwood C, Ellis JG. Group vs. Individual Treatment for Acute Insomnia: A Pilot Study Evaluating a "One-Shot" Treatment Strategy. Brain Sci 2016;7:1.

8. Altena E, Baglioni C, Espie CA, et al. Dealing with sleep problems during home confinement due to the COVID-19 outbreak: Practical recommendations from a task force of the European CBT-I Academy. J Sleep Res 2020. [Epub ahead of print].

9. Baker JG, Sovani M. Case for continuing community NIV and CPAP during the COVID-19 epidemic. Thorax 2020;75:368.

10. Craig S, West $S$ on behalf of the OSA Alliance. Guidance regarding coronavirus (COVID-19) and Obstructive Sleep Apnoea (OSA): for people who routinely use continuous positive airway pressure (CPAP), their families and health care workers. British Thoracic Society. Available online: https://www.brit-thoracic.org.uk/media/455098/osaalliance-cpap-covid-19-advice-20-3-20-v10.pdf

11. Barker J, Oyefeso O, Koeckerling D, et al. COVID-19: community CPAP and NIV should be stopped unless medically necessary to support life. Thorax 2020;75:367.

12. Ebrahim IO, Shapiro CM, Williams AJ, et al. Alcohol and sleep I: effects on normal sleep. Alcohol Clin Exp Res 2013;37:539-49.

13. Zachariae R, Lyby MS, Ritterband LM, et al. Efficacy of internet-delivered cognitive-behavioral therapy for insomnia - A systematic review and meta-analysis of randomized controlled trials. Sleep Med Rev 2016;30:1-10.

14. Steier J, Moxham J. The load and capacity model of healthcare delivery: considerations for the crisis 
management of the COVID-19 pandemic. J Thorac Dis 2020;12:3022-30.

15. Durrant S, Quinnell T, Hare A, et al. BSS Statement on Sleep-Related Advice during the Coronavirus (Covid-19) Pandemic. British Sleep Society. Available online: https://www.sleepsociety.org.uk/wp-content/ uploads/2020/04/BSS-statement-on-sleep-relatedadvice-during-the-coronavirus-Covid-19-pandemi.pdf, Version 1.7; 16-04-2020.

Cite this article as: Steier J, Durrant S, Hare A; on behalf of the BSS Executive Committee. British Sleep Society: the COVID-19 pandemic response. J Thorac Dis 2020;12(8):44694475. doi: $10.21037 /$ jtd-2020-059 